\title{
Risk and Protective Factors Associated with Alcohol, Cigarette, and Marijuana Use During Adolescence
}

\author{
Kelly N. Graves, ${ }^{1}$ Maria E. Fernandez,${ }^{2}$ Terri L. Shelton, ${ }^{3}$ James M. Frabutt, ${ }^{4}$ \\ and Amanda P. Williford ${ }^{5}$
}

Received January 26, 2004; revised June 14, 2004; accepted June 28, 2004

The purpose of this study was to increase the knowledge base of adolescent substance use by examining the influences of risk and protective factors for specific substance use, namely alcohol, cigarettes, and marijuana. Participants included 271 adolescents and their primary caregivers referred for mental health services across North Carolina. A series of hierarchical multiple regressions showed that the relative influences of risk and protective factors differed depending on the target substance in some cases. History of parental felony predicted use of all 3 substances, although the direction of association was substance specific. Parental behavioral control (how families express and maintain standards of behavior) was predictive only of cigarette and marijuana use, not alcohol use. The different links among risk factors, protective factors, and specific substance use are discussed, and recommendations for both mental health and substance use professionals are offered.

KEY WORDS: adolescence; substance use; drug use; marijuana; cigarettes; alcohol.

Adolescents and families who enroll in mental health services often present with a variety of needs above and beyond mental health needs that require treatment. Unfor-

\footnotetext{
${ }^{1}$ Department of Psychology, University of North Carolina at Greensboro, Greensboro, North Carolina. She received her M.A. in Psychology from Wake Forest University and is currently a doctoral student in Clinical Psychology at the University of North Carolina at Greensboro. Her major research interests include developmental pathways to aggressive behavior among females. To whom correspondence should be addressed at Department of Psychology, University of North Carolina at Greensboro, Greensboro, North Carolina; e-mail: knrogers@uncg.edu.

${ }^{2}$ Division of Mental Health, Developmental Disabilities, and Substance Abuse Services. An evaluator for the North Carolina Department of Health and Human Services, her major research interests include system of care intervention programming.

${ }^{3}$ Director, Center for Youth, Family, and Community Partnerships, University of North Carolina at Greensboro. She received her PhD in Clinical Psychology from Purdue University. Her major research interests include developmental psychopathology and early intervention.

${ }^{4}$ Center for Youth, Family, and Community Partnerships, University of North Carolina at Greensboro. His research interests focus on youth violence and youth involved with the juvenile justice system.

${ }^{5}$ Center for Youth, Family, and Community Partnerships, University of North Carolina at Greensboro. She received her $\mathrm{PhD}$ in Clinical Psychology from the University of North Carolina at Greensboro. Her research interests focus on early intervention with young children.
}

tunately, mental health professionals often focus exclusively on the identified mental health problem behaviors without considering other aspects of adolescents' lives that might be related to such behaviors. One such aspect that commonly goes unapprised in mental health assessment and treatment planning is substance use. Research indicates a strong positive relationship between mental illness and substance use among adolescents (Greenblatt, 2000; White et al., 1993, 1999). However, little is known about the risk and protective factors that might be linked differentially to specific types of substances such as alcohol, cigarettes, and marijuana. Therefore, the present study investigates whether various risk and protective factors are differentially related to the frequency of use of 3 specific substances (alcohol, cigarettes, and marijuana) within a clinical sample of adolescents with serious emotional disturbance.

Because substance use "undermines motivation, interferes with cognitive processes, contributes to debilitating mood disorders ... increases risk of accidental injury or death," and contributes to several major health problems such as lung cancer and AIDS (Hawkins et al., 1992, p. 64), the importance of identifying risk and protective 
factors of substance use cannot be overemphasized. In 1996, the Institute of Medicine issued a report that highlighted the gaps in the current substance use literature and recommended areas for future research. One of the top recommendations of that report was to expand the research base regarding the etiology of drug use disorders, including risk and protective factors related to substance use both for specific populations (e.g., White adolescents vs. Black adolescents) and for specific developmental periods (e.g., adolescence). However, the relative influences of risk and protective factors for specific substances such as alcohol, cigarettes, or marijuana remain unclear. The lack of such knowledge poses a major threat to the possible effectiveness of mental health treatment and individualized service planning. Therefore, consistent with previous research focusing mostly on alcohol, cigarettes, and marijuana (e.g., Hawkins et al., 1992; Madu and Matla, 2003), those 3 substances were identified as the targets for the current study.

The consequences of substance use can influence multiple domains in the lives of adolescents. At the individual level, adolescent substance use can interfere with motivation and thinking processes, increase the risk of accidental death and violent crimes, and increase the risk of serious medical complications (Hawkins et al., 1992). For families, adolescent substance use can be destructive to family functioning via increased parental stress, loss of work productivity and income, and a weakening of parent-child bonds. For societies, adolescent substance use quickly depletes community resources and is extremely costly in terms of health care, mental health and drug treatment services, and juvenile crime rates. In fact, it is estimated that the total economic cost of alcohol and drug abuse (including treatment, prevention, health care, and crime) is more than $\$ 240$ billion annually (Martin, 2001). With such widespread consequences, the urgent need to identify risk and protective factors related to the use of alcohol, cigarettes, and marijuana is clear. What remains unclear, however, is whether the risk and protective factors differ in terms of the magnitude of their association depending on the target substance, a question that the present study will attempt to answer.

Thus, the purpose of the present study is to extend research on the links between risk and protective factors for specific substances, namely alcohol, cigarettes, and marijuana. Based on previous research (e.g., Brook et al., 1999; Wagner et al., 2002), it was hypothesized that, (a) older adolescents would report higher frequency of alcohol, cigarette, and marijuana use compared to younger adolescents, (b) male adolescents would report higher frequency of alcohol, cigarette, and marijuana use compared to female adolescents, (c) a history of parental criminal in- volvement would be linked with higher levels of alcohol, cigarette, and marijuana use, (d) a history of parental substance use would be linked with higher levels of alcohol, cigarette, and marijuana use, (e) higher levels of protective factors (i.e., parent-child communication, behavioral control, general family functioning, family involvement, and school functioning) would be linked with lower levels of alcohol, cigarette, and marijuana use, and would be linked most strongly with cigarette and alcohol use due to their overall higher prevalence and frequency rate among adolescents.

\section{METHOD}

\section{Participants}

Participants were a subset of adolescents drawn from the NC FACES (North Carolina Families and Communities Equals Success) grant communities funded by the Center for Mental Health Services in 1997 as part of the Comprehensive Mental Services for Children and Their Families Program. The goal of that nation-wide program was to provide services that are child-centered and family-focused, strengths-based, community-based, and culturally competent. The program also included an evaluation component that assessed system development and individual outcomes for children and families. A full description of the national evaluation protocol and datacollection procedures is provided elsewhere (see Holden et al., 2001).

This cross-sectional study focuses on substance use among adolescents aged 11- to 17-years-old who participated in the outcome study. Over 500 children and their families were enrolled, 376 of whom were at least 11 -years-old. Adolescents who had missing data in relation to either substance use $(n=80)$ or protective factors $(n=25)$ were eliminated. The resulting sample for the present study includes 271 adolescents and their primary caregivers. The present sample $(N=271)$ had about the same mean age $(M=14.50, S D=1.62)$ as those who were excluded from the study $(M=14.00, S D=1.68)$. Distributions of adolescent gender and ethnicity for the current sample also were similar to the total number of adolescents served. Thus, the current sample is assumed to be representative of adolescents and families with serious emotional disturbance in terms of demographic characteristics, at least across North Carolina.

Of the 271 participants, $69 \%$ were male $(31 \%$ female). Forty percent of caregivers identified themselves as Black whereas $54 \%$ identified themselves as White (6\% identified as Hispanic or "Other"). Approximately 
$72 \%$ of adolescents were in the custody of at least 1 biological parent, with $45 \%$ of adolescents living with their mother only, $4 \%$ living with their father only, and 30\% living with both their mother and their father. Of the other legal custodians, $8 \%$ were grandparents, $4 \%$ were adoptive or foster parents, $2 \%$ were other relatives (e.g., aunt or uncle), and $14 \%$ were in state custody (i.e., child welfare services).

Thirty-seven percent of caregivers reported having attended at least some college; $32 \%$ had a high school diploma; and $31 \%$ were not high school graduates. Thirtysix percent of caregivers reported their total family income as being under $\$ 10,000 ; 17 \%$ were between $\$ 10,000$ and $\$ 14,999 ; 25 \%$ were between $\$ 15,000$ and $\$ 24,999$; and $22 \%$ were above $\$ 25,000$. Thus, participants in the current study were primarily of lower socioeconomic status.

All adolescents had at least 1 clinical diagnosis, with 49\% diagnosed with attention deficit-hyperactivity disorder, $21 \%$ diagnosed with a mood disorder, $38 \%$ diagnosed with oppositional defiant disorder, $11 \%$ diagnosed with conduct disorder, $7 \%$ diagnosed with a primary substance use disorder, $4 \%$ diagnosed with a learning disorder and $7 \%$ diagnosed with an anxiety disorder. Fifty-six percent of adolescents had at least 1 co-morbid disorder, with 6\% identified as having a co-morbid substance use disorder.

\section{Procedures}

After being referred to area mental health programs for services, intake workers asked the child and family if they would like to be a part of the outcome study. If the primary caregiver agreed to be contacted, families were informed that an interviewer would be contacting them within a few days to schedule an interview. Interviews were scheduled as soon as possible, but no later than 30 days after the initiation of system of care-based services.

Approximately $96 \%$ of eligible families agreed to participate, and after scheduling the interview, trained evaluators conducted in-home baseline interviews lasting approximately $2 \mathrm{~h}$ for caregivers and $1 \mathrm{~h}$ for adolescents. The higher participation rate can be attributed, at least partly, to the fact that enrollment into the outcome study occurred at the same time as enrollment into service utilization (although participants were assured that they would still be able to receive all services should they decide not to participate in the outcome study). The adolescent and caregiver interview was conducted separately to ensure confidentiality and accurate reporting. Consent forms were read aloud to both the caregiver and adolescent, and questions were answered prior to the start of the interview. Monetary incentives were provided to the respondents depending on the number of instruments that were completed $(\$ 10.00-\$ 30.00)$.

\section{Measures}

\section{Demographic Information and Risk Factors}

The Demographic Information Questionnaire (DIQ; Center for Mental Health Services, 1997a,b) is a 37-item, caregiver-reported measure of a variety of child and family characteristics. Furthermore, the presence of circumstances and conditions in both individual and family domains that previous research has identified as risk factors for emotional and behavioral challenges are ascertained. In addition to basic questions such as the child's gender, age, and ethnicity, caregivers responded either "Yes" or "No" to risk factors including parental history of felony and parental substance use.

Caregiver-reported adolescent functioning was obtained using the Total Problem Index Score from the Child Behavior Checklist (CBCL; Achenbach, 1991). The Total Problem Index is a 113-item index that assesses overall behavioral and emotional problems, and include descriptors such as "Depressed," and "Gets into many fights." Caregivers responded to each item on a 3-point scale, from $0=$ the behavior was not true of the child through through $2=$ the behavior was very true or often true of the child. Internal reliability (>.82), test-retest reliability ( $>.87$ for all scales), and validity have been demonstrated in previous studies (Achenbach, 1991). Higher scores indicated decreased adolescent functioning.

\section{Substance Use}

Substance use was assessed using Part A of the Substance Use Survey (SUS-A; CMHS, 1997a,b). In that questionnaire, adolescents are asked if they ever used any of a list of 12 substances ranging from alcohol to non-prescription drugs, how old they were when they first tried the substance, and how often they used the substance in the past 30 days. For alcohol and marijuana, adolescents were asked, "Within the past 30 days, on how many occasions did you use $(x)$ substance." For cigarettes, adolescents were asked how often they smoked cigarettes on a scale of $1=$ not at all through $7=$ more than 1 pack per day. Because the response scales were different across substances, and because of low variability in the responses, items were transformed to the following scale: $0=$ never used, $1=$ occasional use $(1-2$ times in the last month), and $2=$ frequent use ( 3 or more times in the last month). Additionally, because substance abuse 
research tends to focus mostly on alcohol, cigarettes, and marijuana, and because these substances were the most prevalent in this sample, those 3 substances were identified as the targets for the current study. Higher scores indicated more frequent substance use.

\section{Protective Factors}

Protective factors across various domains were assessed using 2 subscales from the Behavioral and Emotional Rating Scale (BERS; Epstein and Sharma, 1997) that were conceptually related to adolescent substance use. Those 2 subscales were Family Involvement and School Functioning. The Family Involvement subscale is a 10item subscale that assesses the child's participation in family activities, with sample items including, "Over the past 3 months, my child demonstrates a sense of belonging to the family." The School Functioning subscale is a 9-item subscale that assesses the child's functioning in school, with sample items including, "Over the past 3 months, my child attends school regularly." Caregivers responded to each item on a 4-point scale, from $0=$ the behavior was not at all like the child through $3=$ the behavior was very much like the child. Composite scores for each subscale were created by summing all items within that subscale. Internal consistency (Cronbach's alpha) was .84 for the Family Involvement subscale, and .79 for the School Functioning subscale. Higher scores indicated increased positive family involvement and school functioning.

Levels of parent-child communication, behavioral control (how families express and maintain standards of behavior), and general family functioning were reported by adolescents using the Family Assessment Device (FAD; Epstein et al., 1983). Responses are made on a 4-point scale, from $0=$ strongly disagree through $4=$ strongly agree. A total score for each subscale was used in the present study, and internal consistency (Cronbach's alpha) equaled or exceeded .87 for each subscale. The correlation between parent-child communication and behavioral control was .36 $(p<.001)$, the correlation between parent-child communication and general family functioning was $.55(p<.001)$, and the correlation between behavioral control and general family functioning was $.54(p<.001)$. Higher scores indicated higher levels of parent-child communication, behavioral control, and general family functioning.

\section{Statistical Analyses}

To examine the frequency of substance use over the past 30 days, descriptive and frequency analyses were conducted. Additionally, correlational analyses were conducted to examine bivariate associations between variables. A series of 3 hierarchical regression models were used to examine the frequency of alcohol, cigarette, and marijuana use in the past 30 days. Each of the dependent variables was regressed first on demographic variables, followed by risk factors in the 2nd step, and protective factors in the 3rd step.

\section{RESULTS}

Descriptive and frequency analyses are presented in Table I. Those analyses indicated that cigarettes were the most frequently used substance, followed by alcohol and marijuana. Likewise, the highest percentage of adolescents reporting trying cigarettes at least once followed by marijuana and alcohol, respectively. However, although adolescents were most likely to experiment with cigarettes and marijuana (compared to alcohol), adolescents tended to continue to use cigarettes and alcohol compared to marijuana after trying it the first time. The current percentages are similar to other estimates of substance use for SED populations (e.g., Holden and Santiago, 2002), and are significantly higher than the general population for cigarette and marijuana use (but not alcohol use) (US Department of Health and Human Services, 2003). Within the general

Table I. Descriptive and Frequency Statistics for Predictor Variables

\begin{tabular}{|c|c|c|c|c|}
\hline Indicator & $\%$ & Mean & SD & Range \\
\hline Male & 69 & & & \\
\hline White & 54 & & & \\
\hline Black & 40 & & & \\
\hline Used alcohol & $22^{a}$ & .21 & .55 & $0-2$ \\
\hline Used cigarettes & $69^{a}$ & .49 & .81 & $0-2$ \\
\hline Used marijuana & $56^{a}$ & .21 & .58 & $0-2$ \\
\hline Parental history of felony & 52 & & & \\
\hline Parental substance use & 70 & & & \\
\hline Primary substance use disorder & 7 & & & \\
\hline Co-morbid substance use disorder & 6 & & & \\
\hline Identified w/substance use issues & 13 & & & \\
\hline Age & & 14.50 & 1.62 & $11.00-18.00$ \\
\hline Parent-child communication ${ }^{b}$ & & 2.79 & .38 & $1.89-4.00$ \\
\hline Parental behavioral control ${ }^{b}$ & & 3.13 & .40 & $1.78-4.00$ \\
\hline General family functioning $^{b}$ & & 2.84 & .43 & $1.58-3.92$ \\
\hline Family involvement ${ }^{b}$ & & 15.74 & 6.18 & $0.00-30.00$ \\
\hline School functioning $^{b}$ & & 11.23 & 5.97 & $0.00-21.00$ \\
\hline
\end{tabular}

${ }^{a}$ Percentages of adolescents who reported using a substance at least once in the past 30 days.

${ }^{b}$ Higher scores indicate: more communication, behavioral control, better family functioning, greater family involvement and better school functioning. 
Table II. Results of Regression Analyses to Predict Alcohol Use

\begin{tabular}{lrrrrrr}
\hline \multicolumn{1}{c}{ Variables entered } & \multicolumn{1}{c}{$b$} & $t$ & $\beta$ & $F$ & $R^{2}$ & $N$ \\
\hline Block 1 & & & & $13.27^{* * *}$ & .33 & 271 \\
$\quad$ Adolescent age & .05 & $2.17^{*}$ & .18 & & & \\
Adolescent gender & -.09 & -.95 & -.07 & & & \\
Ethnicity & .02 & .43 & .03 & & & \\
$\quad$ Cigarette use & .26 & $3.58^{* * *}$ & .35 & & & \\
$\quad$ Marijuana use & .17 & $1.95^{*}$ & .18 & & & \\
Block 2 & & & & $9.02^{* * *}$ & .35 & \\
$\quad$ Severity of problem Behavior & .01 & .26 & .02 & & & \\
Parental history of felony & -.21 & $-2.02^{*}$ & -.17 & & & \\
$\quad$ Parental history of substance use & -.01 & .09 & .01 & & & \\
Block 3 & & & & $5.77^{* * *}$ & .37 & \\
$\quad$ Parent-child communication & -.10 & -.48 & -.05 & & & \\
Parental behavioral control & -.24 & -1.41 & -.13 & & & \\
General family functioning & .06 & .35 & .04 & & & \\
Family involvement & .01 & .57 & .05 & & & \\
School functioning & .02 & .81 & .08 & & & \\
\hline * $p$ < .05; *** $p<.001$. & & & & & &
\end{tabular}

population of adolescents aged $12-17,28.8 \%$ report ever using alcohol, 26\% report ever smoking cigarettes, and $20.6 \%$ report ever smoking marijuana (US Department of Health and Human Services, 2003).

Frequency of alcohol consumption was correlated positively with frequency of marijuana use over the past 30 days $(r=.53, p<.001)$, and frequency of cigarette use over the past 30 days $(r=.52, p<.001)$. Additionally, frequency of cigarette use over the past 30 days was correlated positively with frequency of marijuana use over the past 30 days $(r=.50, p<.001)$. Thus, adolescents who frequently used 1 type of substance were likely to also use other types of substances. Socioeconomic status was not correlated with alcohol $(r=-.11, n s)$, cigarette $(r=-.04, n s)$, or marijuana use $(r=-.04, n s)$.

\section{Hierarchical Regression Model to Predict Associations With Substance Use}

A series of 3 hierarchical regression models were conducted separately for each substance. Frequency of use for each substance was predicted with several risk factors (i.e., severity of adolescent problem behavior, parental history of substance use, parental history of felony) and protective factors (i.e., parent-child communication, parental behavioral control, family functioning, family involvement, and school functioning). Control variables in all analyses included age, gender, and ethnicity, as well as the 2 substances that were not being predicted (i.e., if predicting alcohol use, cigarette and marijuana use were controlled).
Given the high correlation between substances, it was necessary to confirm that multicollinearity did not present a problem in these analyses. Thus, variance inflation factors (VIF) were examined for each independent variable in each regression. VIF's greater than 10 indicate the presence of problematic multicollinearity (Kleinbaum et al., 1988). All VIF's in the present regressions were equal to or less than 2.49 , indicating the absence of problematic multicollinearity.

\section{Alcohol Use}

The hierarchical regression model to predict frequency of alcohol use in the past 30 days is presented in Table II. When predicting frequency of alcohol use, there was a main effect of adolescent age, with older adolescents reporting more frequent use of alcohol compared to younger adolescents. Consistent with correlational analyses, there also were main effects of both cigarette use and marijuana use, indicating that adolescents were more likely to use alcohol if they also used cigarettes and marijuana. In terms of risk factors, there was a main effect of parental history of felony, with adolescents who reported greater frequency of alcohol use having parents who were less likely to have committed a felony. There were no significant relations among protective factors and alcohol frequency.

\section{Cigarette Use}

The hierarchical regression model to predict frequency of cigarette use in the past 30 days is presented 
Table III. Results of Regression Analyses to Predict Cigarette Use

\begin{tabular}{lrrrrrr}
\hline \multicolumn{1}{c}{ Variables entered } & $b$ & $t$ & $\beta$ & $F$ & $R^{2}$ & $N$ \\
\hline Block 1 & & & & $29.47^{* * *}$ & .52 & 271 \\
$\quad$ Adolescent age & .07 & $2.74^{* *}$ & .19 & & & \\
Adolescent gender & .16 & 1.52 & .10 & & & \\
Ethnicity & .04 & .69 & .04 & & & \\
Alcohol use & .33 & $3.58^{* * *}$ & .25 & & & \\
$\quad$ Marijuana use & .56 & $6.60^{* * *}$ & .45 & & & \\
Block 2 & & & & $19.71^{* * *}$ & .54 & \\
$\quad$ Severity of problem behavior & .00 & .65 & .04 & & & \\
$\quad$ Parental history of felony & .27 & $2.36^{*}$ & .17 & & & \\
Parental history of substance use & -.07 & -.59 & -.04 & & & \\
Block 3 & & & & $12.69^{* * *}$ & .56 & \\
$\quad$ Parent-child communication & .10 & .46 & .04 & & & \\
Parental behavioral control & -.44 & $-2.43^{*}$ & -.18 & & & \\
General family functioning & .11 & .54 & .05 & & & \\
Family involvement & -.01 & -.23 & -.02 & & & \\
School functioning & .02 & .86 & .07 & & & \\
\hline$*$ & & & & & &
\end{tabular}

in Table III. When predicting cigarette use, there was a main effect of adolescent age, with older adolescents reporting greater use of alcohol compared to younger adolescents. Consistent with correlational analyses, there also were main effects of both alcohol use and marijuana use, indicating that adolescents were more likely to smoke cigarettes if they have also used alcohol and marijuana. As with the regression for alcohol, there was a main effect of parental history of felony. However, for cigarettes use, adolescents who reported greater frequency of cigarette use had parents who were more likely to have committed a felony. In terms of protective factors, there was a main effect of parental behavioral control, with higher levels of behavioral control linked with lower frequency of adolescent cigarette use.

\section{Marijuana Use}

The hierarchical regression model to predict marijuana use in the past 30 days is presented in Table IV. When predicting marijuana use, there was a main effect of alcohol and cigarette use, indicating that adolescents were more likely to smoke marijuana if they also used alcohol and cigarettes. Consistent with the finding for alcohol, there was a main effect of parental history of

Table IV. Results of Regression Analyses to Predict Marijuana Use

\begin{tabular}{lrrrrrr}
\multicolumn{1}{c}{ Variables entered } & $b$ & $t$ & $\beta$ & $F$ & $R^{2}$ & $N$ \\
\hline Block 1 & & & & $19.53^{* * *}$ & .42 & 271 \\
$\quad$ Adolescent age & .01 & .47 & .04 & & & \\
Adolescent gender & -.03 & -.32 & -.02 & & & \\
Ethnicity & -.04 & -.81 & -.05 & & & \\
Alcohol use & .16 & $1.94^{*}$ & .15 & & & \\
$\quad$ Cigarette use & .43 & $6.60^{* * *}$ & .54 & & & \\
Block 2 & & & & $14.32^{* * *}$ & .46 & \\
Severity of problem behavior & .01 & .16 & .01 & & & \\
Parental history of felony & -.26 & $-2.65^{* *}$ & -.20 & & & \\
Parental history of substance use & .29 & $2.89^{* *}$ & .21 & & & \\
Block 3 & & & & $10.17^{* * *}$ & .51 & \\
$\quad$ Parent-child communication & -.15 & .82 & .07 & & & \\
Parental behavioral control & -.38 & $-2.44^{*}$ & .19 & & & \\
General family functioning & -.26 & -1.57 & -.15 & & & \\
Family involvement & -.02 & -1.46 & -.12 & & & \\
School functioning & -.01 & -.08 & -.07 & & & \\
\hline
\end{tabular}

${ }^{*} p<.05 ;{ }^{* *} p<.01 ;{ }^{* * *} p<.001$. 
felony, with adolescents who reported greater frequency of alcohol use having parents who were less likely to have committed a felony. There also was a main effect of parental history of substance use, with adolescents reporting more frequent marijuana use if their parents also used substances. In terms of protective factors, there was a main effect of parental behavioral control, with higher levels of behavioral control linked with lower frequency of marijuana use.

\section{DISCUSSION}

The present study examines the degree of association among risk and protective factors for the specific substances of alcohol, cigarettes, and marijuana. The focus of the current study addresses the gap in the literature as described by the Institute of Medicine (1996) regarding the predictive variables for specific drug use. By refining the level of analysis from "substances" to specific types of substances, differential associations with respect to risk and protective factors can be illuminated. That elucidation has the potential to predict more clearly which adolescents are at heightened risk for engagement in specific substance use.

Estimates of substance use among adolescents in the current study differed depending on the target substance. Unlike previous research (Madu and Matla, 2003; Wagner et al., 2002), which implicated alcohol as the most frequently used substance, the current study found that cigarettes were most frequently used by adolescents. Perhaps the addictive ingredients in cigarettes increase the likelihood of frequent use in order to satisfy physiological cravings. Additionally, adolescents who frequently used cigarettes also were more likely to frequently use both alcohol and marijuana. Related, cigarettes have been identified as a "gate-way" drug to other substances such as marijuana. In fact, there is some evidence that cigarette smoking is a significant predictor of the onset of marijuana use (Brook et al., 1999). The National Household Survey on Drug Abuse (2003) reported that almost 60\% of recent marijuana initiates in 1998 and 1999 had used both cigarettes and alcohol prior to using marijuana. Thus, cigarette smoking poses not only an immediate threat to the health of the adolescent, but also as a significant risk factor to the onset of other substances such as marijuana.

Consistent with that research, adolescents in the current study who used 1 type of substance were more likely to also use other types of substances as well. For example, if adolescents reported frequent use of alcohol in the past 30 days, they also tended to report frequent use of cigarettes and marijuana. Furthermore, across all sub- stances, the percentages of adolescents with serious emotional disturbance who reported frequency of substances over the past 30 days were higher than national averages (US Department of Health and Human Services, 2003).

The high frequency of substance use among adolescents with serious emotional disturbance further substantiates the need for early identification of substance use patterns at the point of entry into service systems. Research indicates that the earlier adolescents initiate the use of substances, the greater their risk for becoming involved in a wide variety of other problem behaviors such as aggression and delinquency (Kaplow et al., 2002). Furthermore, the earlier a child begins to use substances, the shorter the length of time it takes to become dependent on that substance (Pepper, 2001). Thus, early identification and treatment from the day the adolescent and family enter a service system can increase the likelihood that substance use, as well as other problem behaviors, can be curtailed.

Although the age of the adolescent predicted the frequency of alcohol and cigarette use, age did not predict the frequency of marijuana use. Thus, younger adolescents were equally likely to use marijuana as older adolescents. The timeframe for onset of specific substances cannot be determined from the present study. However, with the significant correlations among marijuana, alcohol, and cigarette use, the non-significant relation between age and marijuana frequency use might suggest that younger adolescents who frequently use marijuana will also begin using alcohol and cigarettes, an important piece of information for both researchers and clinicians.

In terms of risk factors, the relationship between parental history of felony and frequency of substance use depended on the target substance of interest. For both alcohol and marijuana, adolescents used alcohol and marijuana more frequently if their parents had not committed a felony. In contrast, for cigarette use, the relationship between parental history of felony and cigarette use was positive, indicating that adolescents were more likely to frequently use cigarettes if their parents committed a felony. Thus, previous negative parental behaviors decreased the frequency of adolescent alcohol and marijuana use, but increased the frequency of adolescent cigarette use. It is important to note that it was not known what type of crimes parents committed or if those crimes were influenced by substance use (e.g., impulsive behaviors, driving under the influence, etc.). Perhaps parents who had previous involvement with law enforcement focused more on decreasing the frequency of the more deviant and immediate consequence-provoking substances of alcohol and marijuana in their adolescents compared to the more normative and less "trouble-causing" substance of cigarettes. 
Or, perhaps parental felony is a marker for more general family dysfunction (e.g., lower authoritative parenting) rather than a direct predictor in its own right. Follow-up qualitative interviews with these families would help to illuminate more clearly the link (both directly and/or indirectly) between parental felony and adolescent substance use.

Parental substance use was related only to the frequency of marijuana use among adolescents, with adolescents reporting more frequent marijuana use if their parents also used substances. Of the 3 target substances examined (i.e., alcohol, cigarettes, and marijuana), marijuana is the only illegal substance for adults. Perhaps parental substance use has the largest influence on adolescents via a perception that substance use is acceptable. That is, an adolescent might think that, "if my parents use an illegal substance, then it is okay for me to use illegal substances." Evidence for that possibility has been found by Li et al. (2002) who suggest that behaviors, attitudes, and social influences related to adolescent substance use can change depending on whether parents use substances (however, the Li et al. article did not address specific substance use).

Or, perhaps adolescents whose parents used substances were more willing to disclose their own marijuana use compared to adolescents whose parents did not report a history of substance use. Disclosure of adolescent substance use might not be as intimidating for adolescents whose parents also use substances. It is likely that in a family environment where parents use substances, the adolescent might perceive substance use as more normative, and thus, might be more apt to disclose his/her own experiences with substances.

In terms of protective factors, parental behavioral control had the largest influence on decreasing the frequency on cigarette and marijuana use. That finding is consistent with previous research (e.g., Barber, 1992, 1996; Rogers et al., 2003; Steinberg et al., 1989) indicating that behavioral control is associated with lower levels of externalizing behaviors. However, parent-child communication, parent-child warmth, general family functioning, and family involvement were not related to the frequency of adolescent substance use.

Although past research indicates that males are more likely to use alcohol, cigarettes, and marijuana compared to females (e.g., Brook et al., 1999; Wagner et al., 2002), that was not the case in the current study, with relatively equal frequency rates of substance use among males and females. One possible reason for that inconsistency is that the rates of substance use among females are quickly rising, with $7 \%$ of female adolescents reporting using alcohol in the 1960 s compared to $31 \%$ of female adolescents today
(Martin, 2001). Alternatively, perhaps gender differences in terms of substance use are found within non-clinical populations only. According to Robins et al. (1991), by the time a girl gets referred for treatment, she already demonstrates a variety of maladaptive behaviors (Robins et al., 1991). It is well-established that substance use is correlated positively with other negative behaviors such as aggression. Thus, if a female is exhibiting one form of negative behavior, there is an increased likelihood that she might also exhibit other forms of negative behavior such as substance use. That pattern is consistent with problem behavior theory, a well-supported theory of behavior that claims that rather than an isolated behavior, problem behaviors are embedded in a larger pattern of general problem behavior (Jessor, 1985).

A strength of the present study was the utilization of both risk and protective factors to predict specific substance use. It is noteworthy that both risk and protective factors were linked to the frequency of substance use. Thus, other clinicians and researchers are challenged to include protective factor indicators into their assessment, treatment, or research protocols. By identifying protective factors present within a child and family, efforts can be made to enhance them, ultimately decreasing the child's susceptibility to engage in alcohol, cigarette, or marijuana use.

Because mental health and substance use problems often co-occur, there is a more general need to raise awareness regarding the risk and protective factors that can predict alcohol, cigarette, and marijuana use. From the day adolescents are referred for mental health treatment, possible substance use issues must be identified and integrated with mental health needs and vice versa. Therefore, mental health and substance use professionals are challenged to merge their efforts by working collaboratively rather than in isolation. It is incumbent upon those serving adolescents and families to be responsive, not reactive, to the needs of adolescents and families.

\section{REFERENCES}

Barber, B. K. (1992). Family, personality, and adolescent problem behaviors. J. Marriage Fam. 54: 69-79.

Barber, B. K. (1996). Parental psychological control: Revisiting a neglected construct. Child Dev. 67: 3296-3319.

Brook, J. S., Brook, D. W., Gordon, A. S., Whiteman, M., and Cohen, P. (1990). The psychosocial etiology of adolescent drug use: A family interactional approach. Genet. Soc. Gen. Psychol. Monogr. 116: 113-267.

Brook, J. S., Kessler, R. C., and Cohen, P. (1999). The onset of marijuana use from preadolescence and early adolescence to young adulthood. Dev. Psychopathol. 11: 901-914.

Center for Mental Health Services. (1997a). The Descriptive Information Questionnaire. Unpublished measure. 
Center for Mental Health Services. (1997b). The Substance Use SurveyPart A. Unpublished measure.

Epstein, N. B., Baldwin, L. M., and Bishop, D. S. (1983). The mcmaster family assessment device. J. Marital Fam. Ther. 9: 171-180.

Epstein, M. H., and Sharma, J. (1997). Behavioral and Emotional Rating Scale: A Strength-Based Approach to Assessment. Austin, TX, PRO-ED.

Greenblatt, J. C. (2000). Patterns of alcohol use among adolescents and associations with emotional and behavioral problems. Substance Abuse and Mental Health Services Administration. Rockville, MD.

Hawkins, J. D., Catalano, R. F., and Miller, J. Y. (1992). Risk and protective factors for alcohol and other drug problems in adolescent and early adulthood: Implications for substance abuse prevention. Psychologic. Bull. 112: 64-105.

Holden, E. W., Friedman, R. M., and Santiago, R. L.(2001). Overview of the national evaluation of the comprehensive community mental health services for children and their families program.J. Emotional Behav. Disord. 9: 4-12.

Holden, W., and Santiago, R. (2002). Substance Use Among Children and Youth Referred to Systems of Care. (Issue Brief Vol. 1, Issue 5). Atlanta, GA: National Evaluation of the Comprehensive Community Mental Health Services for Children and Their Families Program.

Institute of Medicine. (1996). Pathways of Addiction: Opportunities in Drug Abuse Research. Washington, DC: National Academy Press.

Jessor, R. (1985). Adolescent problem drinking: Psychosocial aspects and developmental outcomes. Alcohol Drugs Driving 1: 69-96.

Kaplow, J. B., Curran, P. J., and Dodge, K. A. (2001). Child, parent, and peer predictors of early-onset substance use: A multi-site longitudinal study. J. Abnorm. Child Psychol. 30: 199-216.

Keenan, K., Loeber, R., and Green, S. (2000). Conduct disorder in girls: A review of the literature. Clin. Child Fam. Psychol. Rev. 2: 3-19.

Kilpatrick, D. G., Acierno, R., Sauders, B., Resnick, H. S., Best, C. L., and Schnurr, P. (2000). Risk factors for adolescent substance abuse and dependence: Data from a national sample. J. Consult. Clin. Psychol. 68: 19-30.

Kleinbaum, D., Kupper, L., and Muller, K. (1988). Applied Regression Analysis and Other Multivariate Methods. Boston, PWS-Kent.

Li, C., Pentz, M. A., and Chou, C. P. (2002). Parental substance use as a modifier of adolescent substance use risk. Addiction 97: 15371550.

Madu, S. N., and Matla, M. P. (2003). Illicit drug use, cigarette smoking, and alcohol drinking behavior among a sample of high school adolescents in the Petersburg area of the Northern Province, South Africa. J. Adolesc. 26: 121-136.
Martin, S. (2001). Substance abuse is nation's No. 1 health problem, but there is hope. Monit. Psychol. 32: 10-11.

National Household Survey of Drug Abuse. (2003). The NHSDA Report: Characteristics of Marijuana Users. Retrieved March 13, 2003, from the Office of Applied Studies Division of the Substance Abuse and Mental Health Services Administration (SAMHSA) Web site: http://www.samhsa.gov/oas/2k3/newMJ/newMJ.htm.

Pepper, M. (2001). Blamed and Ashamed: The Treatment Experiences of Youth With Co-Occurring Substance Abuse and Mental Health Disorders and Their Families. (Available from the Federation of Families for Children's Mental Health, Alexandria, VA.)

Robins, L. N., Bates, W. M., and O'Neal, P. (1991). Adult drinking patterns of former problem children. In Pittman, D. P., and White, H. R. (Eds.), Society, culture, and drinking patterns reexamined. Alcohol, culture, and social control monograph series. Piscataway, NJ, US, Rutgers Center of Alcohol Studies, pp. 460-479.

Rogers, K. N., Buchanan, C. M., and Winchell, M. E. (2003). Psychological control during adolescence: Links to adjustment in differing parent/adolescent dyads. J. Early Adolesc. 23: 349-383.

Schuck, A. M., and Widom, C. S. (2001). Childhood victimization and alcohol symptoms in females: Causal inferences and hypothesized mediators. Child Abuse Negl. 25: 1069-1092.

Steinberg, L., Elmen, J. D., and Mounts, N. S. (1989). Authoritative parenting, psychosocial maturity, and academic success among adolescents. Child Dev. 60: 1424-1436.

US Department of Health and Human Services. (2001). Report on co-occurring substance abuse and mental health disorders. Retrieved March 6, 2003, from the Substance Abuse and Mental Health Services Administration (SAMHSA) Web site: http://www.samhsa.gov/news/

US Department of Health and Human Services. (2003). National Survey on Drug Use and Health. Retrieved December 12, 2003, from the Substance Abuse and Mental Health Services Administration (SAMHSA) Web site: http://www. samhsa.gov/oas/nhsda/2k2nsduh/Results/2k2Results.htm\#toc

Wagner, E. F., Lloyd, D. A., and Gil, A. G. (2002). Racial/ethnic and gender differences in the incidence and onset age of DSM-IV alcohol use disorder symptoms among adolescents. J. Stud. Alcohol 63: 609-620.

White, H. R., Brick, J., and Hansell, S. (1993). A longitudinal investigation of alcohol use and aggression in adolescents. J. Stud. Alcohol 11: 62-77.

White, H. R., Loeber, R., Stouthamer-Loeber, M., and Farrington, D. (1999). Developmental associations between substance use and violence. Dev. Psychopathol. 11: 785-803. 